\title{
Decadal Advances and Review: Pursuing Space Science and Technology Innovation
}

\author{
Qian Xuesen Laboratory of Space Technology ${ }^{1}$
}

Published online: 10 December 2021

(c) Chinese Society of Astronautics 2021

This special issue is dedicated to Qian Xuesen Laboratory of Space Technology (hereinafter referred to as Qian Lab) on its 10th anniversary. Qian Lab was established in December 2011, in honor of the 100 years birth anniversary of the world-renowned scientist, Dr. Qian Xuesen. As the "Special Innovative Zone" of China Academy of Space Technology, Qian Lab aims to bloom into a world-class laboratory of space science and technology.

Qian Lab remains in the frontier of the transition of the Chinese space industry in the past decade. In the faith to cater the major national needs and the shared welfare of all mankind, Qian Lab has concentrated on: space strategic planning, space system and metasystem demonstrations, engineering sciences, and frontier technologies. Six academicians from the Chinese Academy of Sciences and Chinese Academy of Engineering lead the research, together with over 220 full-time researchers and about 50 students. Facing flourish opportunities of the future space systems and missions, Qian Lab considers itself the bridge between space industry and research in China and abroad. It works vigorously to strengthen close cooperation with partners and forms close bonds. Qian Lab has made a great contribution to the advancement of cutting-edge space science and technology.

Articles within one special issue cannot cover all the research fields involved in Qian Lab. However, this special issue presents what Qian Lab has been always pursuing, devoting to the progression of space science and technology. We elaborately select 5 research articles that cover the specific topics of space quadruped crawling robot, smartmaterials-based flexible actuators, photonics-based superheterodyne, greenhouse gas flux direct calculation method, and satellite formation by electromagnet, that reflect the joint efforts and multidisciplinary approaches of research at Qian Lab.

"How did Nine Spheres divide and join up side by side?", which is asked by ancient Chinese poet Qu Yuan in his masterpiece Heavenly Questions (Tian Wen) thousands of years ago. The question is also raised by the crew of Qian Lab, who strive for a better understanding of our place in the universe, enable future space explorations, and benefit life on Earth. A decade remains just another starting point, and Qian Lab's people will remain dedicated and committed to the development of the Chinese space industry and the well-being of humanity.

We would like to further express our gratitude to Prof. Bao Weimin, Guest Editor of Advances in Astronautics Science and Technology for his cordial support for this special issue. Our gratitude also goes to the whole editorial team for their professionalism and enthusiasm.
Qian Xuesen Laboratory of Space Technology

yiyianyuan@qxslab.cn

1 Beijing, China 